\title{
EVASÃO EM UM CURSO DE PÓS-GRADUAÇÃO A DISTÂNCIA: PERFIL DOS ESTUDANTES DO CURSO DE GESTÃO DA ASSISTÊNCIA FARMACÊUTICA
}

\author{
FLORIANÓPOLIS/SC MAIO/2018
}

\author{
Adriana Cristiane Longo Tristão - UFSC - adriana_cristiane_@hotmail.com \\ Bernd Heinrich Storb - UFSC - bhstorb@yahoo.com.br \\ Fabíola Bagatini Buendgens - UFSC - fabiola_bagatini@yahoo.com.br \\ Mareni Rocha Farias - UFSC - marenif@yahoo.com.br \\ Norberto Rech - UFSC - norberto.rech@terra.com.br \\ Eliana Elisabeth Diehl - UFSC - elianadiehı@hotmail.com
}

Tipo: Investigação Científica (IC)

Natureza: Relatório Final de Pesquisa

Categoria: Pesquisa e Avaliação

Setor Educacional: EDUCAÇÃO CONTINUADA EM GERAL

\begin{abstract}
RESUMO
A qualificação dos trabalhadores do Sistema Único de Saúde (SUS) é fundamental para a qualidade dos serviços prestados. O Curso de Gestão da Assistência Farmacêutica - EaD (GAF-EaD), financiado pelo Ministério da Saúde, foi uma estratégia para capacitação de farmacêuticos no âmbito do SUS. O Curso foi ofertado pela Universidade Federal de Santa Catarina (UFSC) em duas edições (2010 e 2014). A evasão dos estudantes é um dos principais desafios a ser enfrentado e uma série de fatores podem influenciar na decisão do estudante em permanecer ou não em um curso. $O$ objetivo do presente trabalho foi descrever o perfil dos estudantes evadidos em ambas as edições do curso GAFEaD. Os dados utilizados na presente análise foram obtidos a partir do Sistema de Controle Acadêmico da Pós-Graduação da UFSC e foram analisados no software Microsoft Excel®. Consideraram-se evadidos os estudantes que reprovaram ou foram desligados por frequência insuficiente e aqueles que formalizaram a desistência por meio de formulário específico. As variáveis analisadas foram: idade, sexo, Polo Regional Presencial, distância entre o município de residência e o Polo Presencial, tempo de formado (em anos) e vínculo empregatício. Os resultados foram apresentados de forma descritiva, por meio de frequência absoluta e relativa. A taxa de evasão no Curso GAF-EaD, considerando as duas edições, foi de 38,0\%. As características dos estudantes evadidos foram semelhantes em ambas as edições, havendo o predomínio do sexo feminino, faixa etária entre 25 e 39 anos, até 10 anos de formado, residentes mais afastados do Polo Regional, vinculados aos Polos da região Nordeste ou Norte, e estatutários, sendo que algumas dessas características refletem o perfil geral dos estudantes matriculados no Curso. A identificação do perfil de evasão pode contribuir para a definição de estratégias que contribuam para a permanência dos estudantes em cursos a distância, especialmente aqueles de pós-graduação.
\end{abstract}

Palavras-chave: Evasão; Educação a distância; Educação permanente em saúde.

\section{AGRADECIMENTOS}

APOIO FINANCEIRO: FUNDO NACIONAL DE SAÚDE. 


\section{Introdução}

O fortalecimento do Sistema Único de Saúde (SUS), por meio de melhorias e mudanças nas práticas de saúde, está relacionado com transformações na formação e qualificação dos seus trabalhadores. No entanto, dificuldades relacionadas à dimensão geográfica do país, à existência de um elevado número de trabalhadores e gestores, e à diversidade de processos de trabalho demandantes de qualificação exigem criatividade e investimentos em estratégias pedagógicas que alcancem um maior número de trabalhadores do que as formas tradicionais dos cursos presenciais. Nesse sentido, a Educação a Distância $(\mathrm{EaD})$, quando bem planejada, pode tornar-se uma abordagem bastante exitosa, envolvendo o ensino e o serviço, tendo o trabalho como princípio pedagógico, na busca pelo comprometimento, qualidade e eficácia do processo de ensino-aprendizagem (PAIM; GUIMARÃES, 2009).

Apesar das inúmeras vantagens da $\mathrm{EaD}$, a evasão escolar ainda é um dos principais desafios enfrentados pelas Instituições de Ensino Superior (IES) (SILVA, 2013). A evasão resulta de uma série de fatores que influenciam na decisão do estudante em permanecer ou não em um curso. Trata-se de um dos problemas prioritários do sistema de educação que preocupa as IES, pois gera desperdício de recursos financeiros, sociais e humanos (BENTES; KATO, 2014).

As taxas de evasão citadas na literatura variam significativamente, dependendo da fonte e/ou forma de cálculo. Segundo o "Censo EAD.BR: relatório analítico da aprendizagem a distância no Brasil 2016" (ABED, 2017), as taxas de evasão variam principalmente entre $11 \%$ e $25 \%$. Nos cursos regulamentados, as questões financeiras foram apontadas como o principal fator relacionado à evasão. Já nos cursos livres, a falta de tempo foi a causa mencionada com maior frequência.

Revisões de literatura citam para cursos corporativos brasileiros taxa de evasão acima de $30 \%$. Na Ásia há relatos de que mais da metade dos alunos inscritos em programas de educação a distância não concluem seus estudos e na Europa os índices giram em torno de 50\% (ALMEIDA et al., 2013).

Os cursos presenciais, semipresenciais e corporativos contam com mecanismos que vão além do conteúdo e da interação on-line com professores e colegas para atrair e manter o engajamento. Já os cursos regulamentados integralmente a distância e os cursos livres não corporativos dependem totalmente da interação do aluno com o conteúdo e com seus professores e tutores. O investimento na qualidade e variedade dos conteúdos, bem como nos instrumentos de distribuição de conteúdos e interação 
com o professor ou tutor precisa ser muito maior do que quando há outros incentivos para o aluno permanecer (ABED, 2017).

\section{Objetivos}

Descrever o perfil dos estudantes evadidos da primeira e da segunda edições do curso de Gestão da Assistência Farmacêutica - EaD da Universidade Federal de Santa Catarina.

\section{Referência contextual}

A formação na área da saúde está amparada no artigo 200, da Constituição Federal de 1988, em seu inciso III, cuja competência está atribuída ao SUS (BRASIL, 1988). Em 2004 e posteriormente alterada em 2007 (Portaria GM/MS n. 1996, de 20 de agosto de 2007), foi instituída a Política Nacional de Educação Permanente em Saúde como estratégia do SUS para a formação e o desenvolvimento de trabalhadores para o setor (BRASIL, 2009). Uma das ações de educação permanente foi a criação pelo Ministério da Saúde em 2010 da Universidade Aberta do Sistema Único de Saúde (UnA-SUS), visando atender às necessidades de capacitação e educação permanente dos profissionais de saúde, sendo que todos os cursos ofertados pela UnA-SUS são na modalidade de EaD.

O Curso de Gestão da Assistência Farmacêutica - EaD, organizado no âmbito da UnASUS e financiado pelo Ministério da Saúde, por meio da Secretaria de Gestão do Trabalho e da Educação na Saúde (SGTES) foi ofertado em duas edições pela Universidade Federal de Santa Catarina (UFSC). A oferta de vagas foi realizada de modo independente e diferenciada nas duas edições do Curso.

A primeira edição teve início em 2010 e foi desenvolvida em quatro diferentes etapas, de acordo com a região geográfica do país, certificando como Especialização. A primeira etapa foi a Nordeste, seguida das etapas Norte/Centro-Oeste e Sul, finalizando com etapa Sudeste, em 2013. Foram ofertadas 2000 vagas para farmacêuticos atuantes no serviço público de saúde, as quais foram distribuídas de acordo com o número de municípios contidos em cada região, considerando uma relação 2,4 a 3,0 municípios por vaga, em cada etapa. Os critérios de seleção foram regionalizados e pactuados com os gestores estaduais de saúde e os Coordenadores de Polo Regional Presencial, em cada região.

A segunda edição do Curso teve início em 2014, em uma única etapa, na qual foram 
ofertadas três modalidades de certificação (Estudos de Aprofundamento, Aperfeiçoamento e Especialização). Nesta edição, foram oferecidas vagas para farmacêuticos atuantes no serviço público de saúde e para docentes de cursos de graduação em Farmácia, na proporção 3:1, sendo que:

1. Entre as vagas oferecidas para farmacêuticos que atuavam no serviço público de saúde, $80 \%$ foram destinadas a municípios participantes do Programa Nacional de Qualificação da Assistência Farmacêutica (QUALIFAR-SUS) e/ou Projeto de Formação e Melhoria da Qualidade em Rede de Atenção à Saúde (QualiSUSRede) e as 20\% complementares foram destinadas aos demais municípios. Os farmacêuticos com vínculo estadual e/ou federal com atuação na esfera municipal concorreram de acordo com a participação ou não do município nos programas mencionados.

2. Para as vagas oferecidas aos docentes de cursos de Farmácia, foi priorizada uma vaga para cada IES e, em caso de vagas remanescentes, poderiam ser classificados até mais três docentes para a mesma IES. Para as IES públicas (municipal, estadual ou federal), cada campi foi considerado uma IES distinta; já para as privadas e especiais, cada IES teria, no máximo, três vagas, independentemente do número de campi.

Em ambas as edições, o número de estudantes matriculados foi superior ao número de vagas inicialmente ofertado, já considerando a evasão prevista. O total de inscritos nas duas edições foi de 9.866 profissionais e docentes, o que demonstra a acentuada demanda por qualificação na área de Assistência Farmacêutica no SUS. Ao final, 2.500 profissionais e docentes concluíram o curso, sendo que 2.472 receberam o título de Especialista em Gestão da Assistência Farmacêutica; 12, o certificado de Estudos de Aprofundamento; e 16, de Aperfeiçoamento.

A modalidade EaD demonstra uma trajetória consistente no cenário internacional e, seguindo a mesma tendência, também no contexto nacional. Atualmente, desfruta de maior credibilidade, sendo reconhecida como uma modalidade tão eficaz quanto a presencial. Apesar de a EaD fundamentar a proposta de ampliação e democratização da educação, estudar e ensinar a distância não são tarefas fáceis aos atores diretamente envolvidos, pois tanto alunos quanto professores, precisam passar por um processo de mudança cultural (ALMEIDA et al., 2013).

Segundo Comarella (2009), a evasão pode ser definida como a saída do estudante de um curso ou do sistema de educação no qual está matriculado, sem concluí-lo com sucesso. Martinez (2003) classifica os tipos de evasão na EaD como: Dropout (evasão) 
ocorre quando o estudante abandona o curso ou o sistema de educação durante o seu desenvolvimento e nunca retorna; Stopout (trancamento) é a interrupção temporária do curso; e atteainer (evasão do curso) ocorre quando o estudante sai do curso antes da sua conclusão, mas com a aquisição do conhecimento, ou por ter atingido suas metas pessoais. Já o caso dos estudantes que nem chegam a iniciar o curso é chamado de non-starter (não iniciante).

\section{Procedimentos metodológicos}

Trata-se de um estudo transversal, que analisou o perfil dos evadidos nas duas edições do curso Gestão da Assistência Farmacêutica -EaD.

Os dados utilizados na presente análise foram obtidos a partir do Sistema de Controle Acadêmico da Pós-Graduação da UFSC. Os dados foram transportados e analisados no editor de planilhas Microsoft Office Exce®, versão 2010.

Consideraram-se evadidos os estudantes que reprovaram ou foram desligados por frequência insuficiente e aqueles que formalizaram a desistência por meio de formulário específico.

As variáveis analisadas foram: idade, sexo, Polo Regional Presencial, distância do município em que reside do Polo Presencial, tempo de formado (em anos) e vínculo empregatício.

Os resultados foram apresentados de forma descritiva, por meio de frequência absoluta e relativa.

A pesquisa foi aprovada pelo Comitê de Ética em Pesquisa com Seres Humanos da Universidade Federal de Santa Catarina, sob o Certificado de Apresentação para Apreciação Ética (CAAE) n. 46912815.0.0000.0121.

\section{Apresentação e discussão dos resultados}

A primeira edição do Curso de Gestão da Assistência Farmacêutica - EaD teve 2.476 estudantes matriculados, sendo que destes, 983 evadiram, correspondendo a uma taxa de evasão de 39,7\% (Tabela 1).

Na segunda edição do Curso, ofertada em três modalidades, 1.821 estudantes foram matriculados nas três modalidades, sendo que destes, 649 evadiram, correspondente a 
$35,6 \%$ de evasão. A modalidade onde houve maior número de estudantes desistentes e reprovados por frequência insuficiente foi a de Aperfeiçoamento, com 61\% (Tabela 1).

Tabela 1 - Distribuição por situação dos estudantes evadidos na primeira e na segunda edições do Curso de G estão da Assistência Farm acêutica $-E$ aD ( $n=983$ e $n=649$, respectivam ente).

\begin{tabular}{|c|c|c|c|c|c|c|c|c|}
\hline \multirow{3}{*}{ Situação } & \multicolumn{2}{|c|}{$1^{s}$ ed ição } & \multicolumn{6}{|c|}{$2^{a}$ edição } \\
\hline & \multirow[b]{2}{*}{$n$} & \multirow[b]{2}{*}{$\%$} & \multicolumn{2}{|c|}{ Aprofundamento } & \multicolumn{2}{|c|}{ Aperfeiçoamento } & \multicolumn{2}{|c|}{ Especialização } \\
\hline & & & $n$ & $\%$ & $n$ & $\%$ & $n$ & $\%$ \\
\hline Desistente & 172 & $17 \%$ & 10 & $2 \%$ & 105 & $16 \%$ & 15 & $2 \%$ \\
\hline Reprovado por Fl & 757 & $77 \%$ & 50 & $8 \%$ & 295 & $45 \%$ & 174 & $27 \%$ \\
\hline Total: & \multicolumn{2}{|c|}{983} & 60 & $10 \%$ & 400 & $61 \%$ & 189 & $29 \%$ \\
\hline
\end{tabular}

Fonte: Elaborado pela autora, a partir dos dados obtidos.

Legenda: $\mathrm{FI}=$ fequência insuiciente; $n=$ número.

A distribuição por sexo dos estudantes evadidos foi semelhante na primeira e segunda edições, sendo que $66 \%$ e $68 \%$, respectivamente, eram do sexo feminino, conforme Figura 1.

Figura 1 - Distribuição por sexo dos estudantes evadidos na primeira e na segunda edições do Curso de Gestão da Assistência Farmacêutica - EaD (n=983 e n=649, respectivamente).

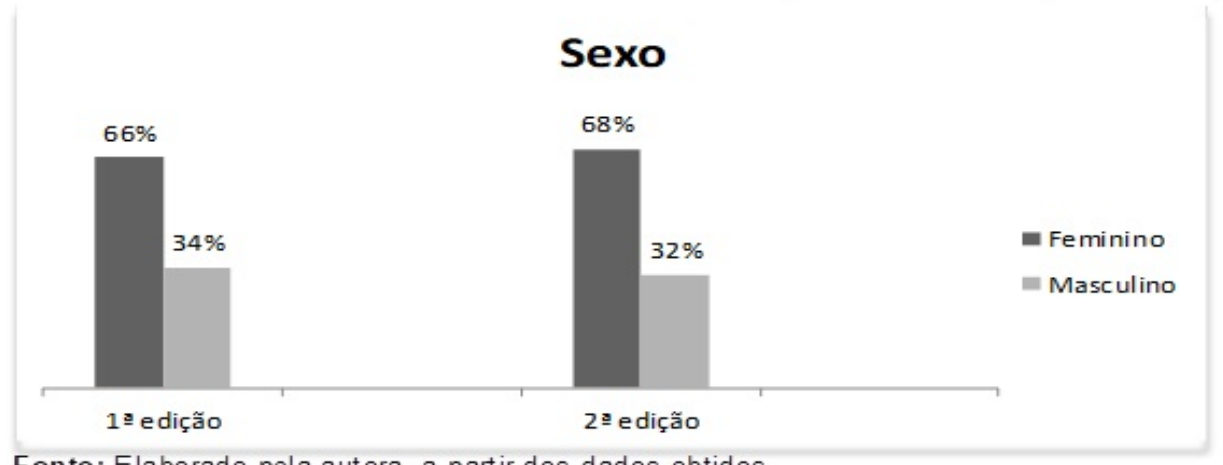

Fonte: Elaborado pela autora, a partir dos dados obtidos.

A faixa etária predominante entre os estudantes evadidos foi entre 25 e 39 anos, em ambas as edições, com valores de 69\% e 77\%, respectivamente, conforme Tabela 2.

Tabela 2 - Distribuição por faixa etária dos estudantes evadidos na primeira e na segunda edições do Curso de Gestão da Assistência Farmacêutica - EaD ( $n=983$ e $n=649$, respectivamente).

\begin{tabular}{|c|c|c|c|c|}
\hline \multirow{3}{*}{ Faixa Etária } & \multicolumn{4}{|c|}{ Edição } \\
\hline & \multicolumn{2}{|c|}{$1^{a}$ e dição } & \multicolumn{2}{|c|}{$2^{\mathrm{a}}$ edição } \\
\hline & $n$ & $\%$ & $n$ & $\%$ \\
\hline Até 24 anos & 55 & $6 \%$ & 26 & $4 \%$ \\
\hline Entre 25 e 39 anos & 683 & $69 \%$ & 498 & $77 \%$ \\
\hline Entre 40 e 59 anos & 239 & $24 \%$ & 122 & $19 \%$ \\
\hline Acima de 60 anos & 5 & $1 \%$ & 3 & $0 \%$ \\
\hline Ignorado & 1 & $0 \%$ & 0 & $0 \%$ \\
\hline
\end{tabular}

Fonte: Elaborado pela autora, a partir dos dados obtidos.

Legenda: $n=$ número.

Quanto ao tempo de formação, na segunda edição a maioria dos alunos evadidos havia se formado em até 5 anos, e na primeira edição houve predomínio de estudantes formados entre 6 a 20 anos (Tabela 3). 
Tabela 3 - Distribuição por tempo de formação dos estudantes evadidos na primeira e na segunda edições do Curso de Gestão da Assistência Farmacêutica - EaD ( $n=983$ e n=649, respectivamente).

\begin{tabular}{|c|c|c|c|c|}
\hline \multirow{3}{*}{ Tempo de formação } & \multicolumn{4}{|c|}{ Edição } \\
\hline & \multicolumn{2}{|c|}{$1^{a}$ e dição } & \multicolumn{2}{|c|}{$2^{\mathrm{a}}$ e dição } \\
\hline & $n$ & $\%$ & $n$ & $\%$ \\
\hline Até 5 anos & 261 & $27 \%$ & 255 & $39 \%$ \\
\hline 6 a 10 anos & 300 & $31 \%$ & 213 & $33 \%$ \\
\hline 11 a 20 anos & 282 & $29 \%$ & 144 & $22 \%$ \\
\hline 21 a 30 anos & 107 & $11 \%$ & 25 & $4 \%$ \\
\hline 31 a 40 anos & 33 & $3 \%$ & 12 & $2 \%$ \\
\hline
\end{tabular}

Fonte: Elaborado pela autora, a partir dos dados obtidos.

Legenda: $n=$ número.

A distância entre o Polo Presencial e a residência do estudante demonstrou que a maioria dos estudantes evadidos residiam a uma distância superior a $50 \mathrm{Km}$ do Polo, conforme Tabela 4, o que pode ter dificultado o comparecimento nos encontros presenciais, para a realização das avaliações e da defesa do Trabalho de Conclusão de Curso (TCC). Salienta-se que conforme o Decreto n. 5.622 de 19 de dezembro de 2005, as avaliações presenciais e a defesa de TCC obrigatoriamente devem ser realizadas na sede da instituição de ensino, nos polos de educação a distância ou em ambiente profissional, conforme as Diretrizes Curriculares Nacionais (BRASIL, 2005). Ao longo da primeira edição houve três encontros presencias e na segunda, quatro para os que optaram pela Especialização.

Tabela 4 - Distribuição por distância, em quilômetros $(\mathrm{Km})$, entre o Polo Regional Presencial e o município de residência dos estudantes evadidos na primeira e na segunda edições do Curso de Gestão da Assistência Farmacêutica $-\mathrm{EaD}$ ( $n=983$ e $n=649$, respectivamente).

\begin{tabular}{|c|c|c|c|c|}
\hline \multirow{3}{*}{$\begin{array}{c}\text { Distância do Polo } \\
\text { Presencial }\end{array}$} & \multicolumn{4}{|c|}{ Edição } \\
\hline & \multicolumn{2}{|c|}{$1^{\mathrm{a}}$ ediçăo } & \multicolumn{2}{|c|}{$2^{\mathrm{a}}$ edição } \\
\hline & $n$ & $\%$ & $n$ & $\%$ \\
\hline $0 \mathrm{Km}$ & 227 & $23 \%$ & 146 & $22 \%$ \\
\hline Até $50 \mathrm{Km}$ & 94 & $10 \%$ & 28 & $4 \%$ \\
\hline 51 a $100 \mathrm{Km}$ & 78 & $8 \%$ & 19 & $3 \%$ \\
\hline 101 a $200 \mathrm{Km}$ & 175 & $18 \%$ & 93 & $14 \%$ \\
\hline 201 a $300 \mathrm{Km}$ & 128 & $13 \%$ & 83 & $13 \%$ \\
\hline 301 a $400 \mathrm{Km}$ & 99 & $10 \%$ & 71 & $11 \%$ \\
\hline 401 a $500 \mathrm{Km}$ & 39 & $4 \%$ & 60 & $9 \%$ \\
\hline 501 a $1.000 \mathrm{Km}$ & 109 & $11 \%$ & 115 & $18 \%$ \\
\hline Acima de $1.000 \mathrm{Km}$ & 34 & $3 \%$ & 34 & $5 \%$ \\
\hline
\end{tabular}

Fonte: Elaborado pela autora, a partir dos dados obtidos.

Legenda: $n=$ número.

Os Polos Presenciais em que mais estudantes evadiram na primeira edição, foram os de Teresina/PI, São Luís/MA, Salvador/BA e Recife/PE, respectivamente, sendo que todos Polos pertenciam à primeira etapa (Nordeste). Na segunda edição, houve maior evasão no Polo Manaus/AM, seguido de Salvador/BA e Vitória da Conquista/BA (Tabela 5). 
Tabela 5 - Dis tribuição da taxa de evas ão por Polo Presencial na primeira e na segunda edições do Curs o de Gestăo da Ass is tência Farmacêutica - EsD ( $n=983$ e $n=649$, res pectivemente).

\begin{tabular}{|c|c|c|c|c|c|c|c|}
\hline \multicolumn{8}{|c|}{ Taxa de Ev asão por Polo Presencial } \\
\hline & $1^{\top}$ edição & & & & $2^{3}$ edição & & \\
\hline Belém / PA & 102 & 44 & $43 \%$ & Brasilia / DF & 126 & 45 & $36 \%$ \\
\hline Goiânia / GO & 161 & 67 & $42 \%$ & Divinópolis / MG & 199 & 52 & $26 \%$ \\
\hline Joirwille / SC & 196 & 61 & $31 \%$ & Fortaleza / CE & 168 & 49 & $29 \%$ \\
\hline Juiz de Fora/MG & 164 & 48 & $29 \%$ & Goiânia / GO & 104 & 37 & $36 \%$ \\
\hline Natal / RN & 130 & 61 & $47 \%$ & Porto Alegre / RS & 97 & 37 & $38 \%$ \\
\hline Niterói / RJ & 135 & 53 & $39 \%$ & Ribeirão Preto / SP & 119 & 40 & $34 \%$ \\
\hline Porto Alegre / RS & 191 & 83 & $43 \%$ & Salvador / BA & 180 & 78 & $43 \%$ \\
\hline Recife / PE & 175 & 90 & $51 \%$ & São Luis / MA & 154 & 56 & $36 \%$ \\
\hline Ribeirão P reto / SP & 189 & 74 & $39 \%$ & São Paulo / SP & 169 & 48 & $28 \%$ \\
\hline Salvador / BA & 169 & 95 & $56 \%$ & Vitória da Conquista / BA & 92 & 40 & $43 \%$ \\
\hline Vila Velha / ES & 135 & 31 & $23 \%$ & & & & \\
\hline Vitória da Conquista / BA & 56 & 24 & $43 \%$ & & & & \\
\hline
\end{tabular}

Fonte: Elsborado pels autora, a partir dos dados obtidos.

Quanto ao vínculo empregatício, conforme Tabela 6, na segunda edição 5\% dos evadidos eram docentes; em relação aos farmacêuticos, $48 \%$ eram estatutários e $17 \%$ eram contratados temporariamente. Na primeira edição, a maior parte era estatutário $(68 \%)$.

Tabela 6 - Distribuição por tipo de vínculo dos estudantes evadidos na primeira e na segunda edições do Curso de Gestão da Assistência Farmacêutica - $\mathrm{EaD}$ ( $\mathrm{n}=983$ e $n=649$, respectivamente).

\begin{tabular}{|c|c|c|c|c|c|}
\hline \multicolumn{6}{|c|}{ Edição } \\
\hline \multicolumn{3}{|c|}{$1^{\text {a }}$ edição } & \multicolumn{3}{|c|}{$2^{\mathrm{a}}$ edição } \\
\hline Vínculo & $n$ & $\%$ & Vínculo & $n$ & $\%$ \\
\hline Farmacêuticos & & & Docente & 31 & $5 \%$ \\
\hline Cargo em comissão & 34 & $3 \%$ & Farmacêuticos & & \\
\hline Celetista & 99 & $10 \%$ & Cargo em comissão & 63 & $10 \%$ \\
\hline Estatutário & 673 & $68 \%$ & Celetista & 80 & $12 \%$ \\
\hline Prestador de serviço & 56 & $6 \%$ & Contratos temporários & 110 & $17 \%$ \\
\hline \multirow[t]{2}{*}{ Temporario } & 121 & $12 \%$ & Estatutário & 309 & $48 \%$ \\
\hline & & & Prestador de Serviço & 56 & $9 \%$ \\
\hline Total & 983 & $100 \%$ & Total & 649 & $100 \%$ \\
\hline
\end{tabular}

Fonte: Elaborado pela autora, a partir dos dados obtidos

Legenda: $\mathrm{n}=$ número

\section{Considerações finais}

A taxa de evasão no Curso de Gestão da Assistência Farmacêutica - EaD, considerando as duas edições, foi de 38,0\% ( $n=1.632)$. As características dos estudantes evadidos foram semelhantes em ambas as edições, havendo o predomínio do sexo feminino, faixa etária entre 25 e 39 anos, até 10 anos de formado, residentes mais afastados do Polo Regional, vinculados aos Polos da região Nordeste ou Norte, e estatutários. 
É importante destacar que algumas dessas características refletem o perfil geral dos estudantes matriculados considerando as variáveis sexo, faixa etária, tempo de formado (em anos), distância do município em que reside do Polo Presencial (em Km) e tipo de vínculo empregatício.

Identificar o perfil dos estudantes que evadiram no Curso de Gestão da Assistência Farmacêutica - EaD, associando aos grupos com maior percentual de evasão, permite traçar estratégias que podem contribuir para a permanência em cursos a distância, especialmente aqueles de pós-graduação.

\section{Referências}

ALMEIDA, O. C. D. S. D. et al. Evasão em cursos a distância: fatores influenciadores. Revista Brasileira de Orientação Profissional, v. 14, n.1, p.19-33, 2013.

ASSOCIAÇÃO BRASILEIRA DE EDUCAÇÃO A DISTÂNCIA. Censo EAD.BR: Relatório Analítico de Aprendizagem a Distância no Brasil 2016 = Censo EAD.BR: analytic report of distance learning in Brazil 2016 / [traduzido por Maria Thereza Moss de Abreu]. Curitiba: InterSaberes, 2017. Disponível em: < http://abed.org.br/censoead2016/Censo_EAD_2016_portugues.pdf> Acesso em 19 maio. 2018.

BENTES, M. C. B.; KATO, O. M. Fatores que afetam a evasão na educação a distância: curso de administração. Revista Psicologia da Educação, São Paulo, v. 39, p. 31-45, 2014.

BRASIL. Constituição (1988). Constituição da República Federativa do Brasil. Brasília, DF: Senado Federal: Centro Gráfico, 1988. 292 p.

BRASIL. Ministério da Saúde. Secretaria de Gestão do Trabalho e da Educação na Saúde. Departamento de Gestão da Educação em Saúde. Política Nacional de Educação Permanente em Saúde. Brasília: Ministério da Saúde, 2009.

BRASIL. Presidência da República. Casa Civil. Decreto n 5.622 de 19 de dezembro de 2005. Regulamenta o art. 80 da Lei o 9.394, de 20 de dezembro de 1996, que estabelece as diretrizes e bases da educação nacional. Diário Oficial [da\} União, Poder Executivo, Brasília, DF, 2005.

COMARELLA, R. L. Educação superior a distância: evasão discente. 2009. 146 f. 
Dissertação (Mestrado em Engenharia e Gestão do Conhecimento) - Centro Tecnológico, Universidade Federal de Santa Catarina, Florianópolis, 2009.

MARTINEZ, M. High attrition rates in e-learning: challenges, predictors, and solutions. The Elearning Developers' Journal, 2003. Disponível em: <https://www.elearningguild.com/pdf/2/071403mgt-I.pdf>

PAIM, M. C.; GUIMARÃES, J. M. M. Importância da formação de docentes em EaD no processo de educação permanente para trabalhadores do SUS na Bahia. Revista Baiana de Saúde Pública, v.33, n.1, p. 94-103, 2009.

SILVA, G. P. Análise de evasão no ensino superior: uma proposta de dignóstico de seus determinantes. Revista Avaliação, Campinas, v. 18, n. 2, p.311-333, 2013. 